\title{
High-Performance Wireless via the Merger of Cl Chip-Shaped DS-CDMA and Oscillating-Beam Smart Antenna Arrays
}

\author{
Seyed Alireza Zekavat \\ Department of Electrical and Computer Engineering, Michigan Technological University, Houghton, MI 49931, USA \\ Email:rezaz@mtu.edu
}

\author{
Carl R. Nassar \\ Department of Electrical and Computer Engineering, Colorado State University, Fort Collins, CO 80523-1373, USA \\ Email:carln@colostate.edu
}

\author{
Steve Shattil \\ Idris Communications, 1500 Cherry St. Suite L, Louisville, CO 80027, USA \\ Email: steve@ciansystems.com
}

Received 29 May 2003; Revised 15 October 2003

\begin{abstract}
We introduce a novel merger of direct sequence code division multiple access (DS-CDMA) and smart antenna arrays. With regard to the DS-CDMA scheme, we employ carrier interferometry DS-CDMA (CI/DS-CDMA), a novel implementation of DSCDMA where chips are decomposable into $N$ narrowband frequency components. With regard to the antenna array, we deploy the oscillating-beam smart array. Here, applying proper time-varying phases to the array elements, we create small movement (oscillation) in the antenna array's pattern, while steering the antenna pattern main lobe to the position of the intended user. The oscillating antenna pattern creates a time-varying channel with a controllable coherence time. This, in turn, provides transmit diversity in the form of a time diversity gain at the mobile receiver side. At the receiver, three stages of combining are available: combining time components of the received signal within symbol duration $T_{S}$ (each experiencing a different fade) to enhance performance via time diversity; combining frequency components which make up the CI/DS-CDMA chip to enhance the performance via frequency diversity; and combining across chips to eliminate the interfering users on the system. Merging CI/DS-CDMA with the oscillating-beam smart antenna at the base station, we achieve very high capacity via the merger of SDMA (available through directionality of the antenna array) and code division multiple access (inherent in CI/DS-CDMA), and very high performance via the construction of receivers that exploit both transmit diversity and frequency diversity. We present the performance gains of the proposed merger.
\end{abstract}

Keywords and phrases: smart antennas, antenna arrays, DS-CDMA systems, transmit diversity, carrier interferometry.

\section{INTRODUCTION}

Antenna arrays located at the base station (BS) enhance wireless communication systems via (1) directionality, which supports space division multiple access (SDMA); or (2) more recently, a transmit diversity benefit, that is, a diversity scheme that uses the antenna array at the BS to exploit diversity at the mobile (see, e.g., $[1,2]$ ). Recently, the authors have introduced a new antenna array scheme in $[3,4,5,6]$ which offers both (1) high capacity via SDMA and (2) excellent probability-of-error performance at the mobile via its transmit diversity benefits. Both benefits are available while maintaining low mobile receiver complexity.
In the authors' proposed antenna array of $[3,4,5,6]$, a unique, carefully controlled time-varying phase shift is applied to each antenna array element, sweeping the beam pattern directed to the mobile such that (1) the beam pattern maintains a constant large scale fade for the symbol duration $T_{S}$; (2) the beam pattern ensures $L$ independent fades within each $T_{S}$; (3) after each $T_{S}$, the antenna beam returns to its initial position, and sweeps same area of space over $T_{S}$ (leading to an oscillating antenna pattern and easing parameter estimation); (4) the movement of the beam pattern, as a percentage of half-power beamwidth (HPBW), is small, allowing the beam pattern to maintain directionality; and (5) the bandwidth expansion due to beam pattern movement is 
negligible. In this paper, we merge this novel antenna array technique with DS-CDMA systems.

Direct-sequence code division multiple access (DSCDMA) [7] is the world's most popular CDMA architecture. In DS-CDMA, each user's bit is multiplied by a sequence of $N$ chips (short pulses of duration $T_{C}$ ), where each chip has amplitude +1 or -1 . By careful selection of +1 and -1 values (spreading sequences), the receiver can separate users one from another. To enhance performance via path diversity (e.g., [8]), most DS-CDMA systems employ RAKE receivers, which attempt to separate and linearly recombine the multiple paths.

Recently, a novel chip shape referred to as the CI (carrier interferometry) chip shape was introduced to DS-CDMA $[9,10,11,12,13]$. Here, each chip is decomposable into $N$ orthogonal carrier components. As a result, when applying these chip shapes, (1) the DS-CDMA receiver: achieves a frequency diversity benefit (rather than a path diversity benefit) by decomposing chips into carrier components and frequency combining; and (2) the use of frequency combining in place of path combining (as done in RAKE receivers) leads to a significantly improved performance via the ability to avoid interpath interference $[9,10,11,12,13]$.

In this work, we innovatively apply the oscillating-beam antenna arrays of $[3,4,5,6]$ to DS-CDMA systems with CI chip shapes (CI/DS-CDMA) of $[9,10,11,12,13]$. This enables (1) very high capacity via the merger of SDMA (directionality of the antenna array) and CDMA (inherent in CI/DS-CDMA); and (2) very high performance via the construction of receivers that exploit both transmit diversity and frequency diversity. We focus on the performance benefits of the proposed merger.

In this work, we assume carrier frequency $\left(f_{0}\right)$ much larger than system bandwidth (BW) (e.g., $f_{0}>100 \cdot(B W)$ ), a reasonable assumption in today's mobile systems. Hence, the antenna pattern is identical for the entire transmit bandwidth. With this in mind, the CI/DS-CDMA signal is fed into a single $M$-element smart antenna array. By carefully designing the phase shifts applied to antenna array elements, the resulting beam pattern corresponds to an oscillating beam pattern similar to that in $[3,4,5,6]$. This leads to a time-varying channel with a controllable coherence time. The controllable coherence time is used by the mobile to exploit time diversity and enhance performance.

The benchmark for comparison in this work is a CI/DSCDMA system employed in conjunction with a conventional smart antenna array (an antenna array which creates an adaptive beam pattern directed toward the intended user, leading to increased capacity via SDMA, but, unlike the proposed scheme, offers no improvements in the performance of CI/DS-CDMA system). This work highlights the performance benefits that can be achieved by small oscillations in the beam pattern of the smart antenna array.

Receivers are constructed to exploit both the transmit diversity, which corresponds to an induced time diversity provided by the antenna array, as well as the diversity inherent in the CI/DS-CDMA system (an exploitable frequency diversity). Thus, at the receiver, three stages of combining are present: (1) a combining of the time components with different fades (to exploit time diversity), (2) a combining across frequency components (to exploit frequency diversity), and (3) a combining across chips (to eliminate users in a traditional DS-CDMA manner). We can apply the combining first on the frequency components or first on the time components, that is, the first and the second combining stages can be interchanged.

Assuming (a) rich scattering environment (where, up to 7 -fold time diversity is achievable via beam pattern movement $[5,6])$, (b) fully loaded CI/DS-CDMA with a processing gain of $N=32$ (i.e., $\mathrm{K}=32$ orthogonal users are available in the system), and (c) 4-fold frequency diversity over the entire bandwidth, simulation results demonstrate that the proposed system achieves $14 \mathrm{~dB}$ gain over a CI/DS-CDMA system with a conventional smart antenna array at a probability of error of $10^{-3}$. Performance gains are even more impressive when the proposed system is compared to a traditional DS-CDMA system with a conventional smart antenna array. (These performance benefits are, in addition to the usual network capacity gains, provided via SDMA.)

Section 2 introduces the merger of the beam-sweeping smart antenna arrays and CI/DS-CDMA. Section 3 presents receiver structures employing equal gain combining (EGC) across frequency components followed by minimum mean square error combining (MMSEC) across time domain components. Section 4 presents simulated performance results, while Section 5 presents a conclusion.

\section{THE MERGER OF CI/DS-CDMA AND BEAM-SWEEPING ANTENNA ARRAYS}

\subsection{The CI/DS-CDMA system}

In DS-CDMA, considering a binary phase shift keying (BPSK) modulation, a unique time sequence ( $N$ chips, each with amplitude +1 or -1 ) is assigned to each user. Hence, the $k$ th user's data bit, $b_{k}$, is sent as

$$
s_{k}(t)=\operatorname{Re}\left\{b_{k} \cdot C_{k}(t) \cdot e^{j 2 \pi f_{0} t}\right\},
$$

where $b_{k}$ is +1 or $-1, f_{0}$ is the center or carrier frequency, and $C_{k}(t)$ is the $k$ th user spreading code, corresponding to

$$
C_{k}(t)=\sum_{i=0}^{N-1} c_{k}^{i} \cdot h\left(t-i T_{C}\right) \cdot g(t) .
$$

Here, $c_{k}^{i} \in\{-1,+1\}$ is the $i$ th element of user $k^{\prime}$ s spreading code, $T_{C}=T_{S} / N$ is the chip duration, and $g(t)$ is a rectangular waveform limiting the chip shape to duration $T_{S}$. In the proposed CI/DS-CDMA system of $[9,10,11,12,13]$, the chip shape $h(t)$ corresponds to a multicarrier signal. Specifically, the chip shape $h(t)$ is a superpositioning of $N$ narrowband subcarriers equally spaced in frequency by $\Delta f$ :

$$
h(t)=\sum_{n=0}^{N-1} e^{j 2 \pi n \Delta f t}
$$




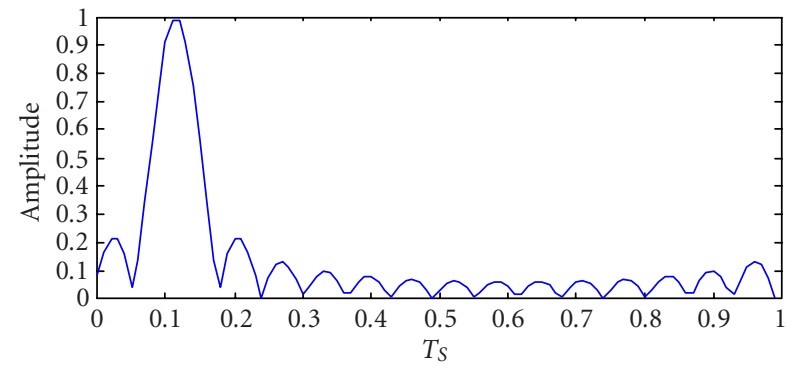

(a)

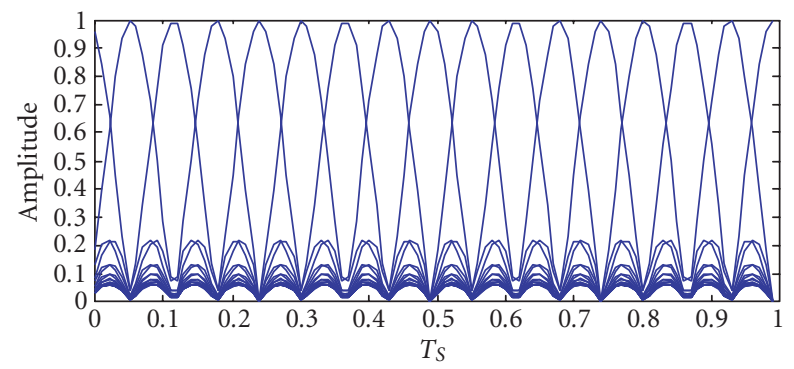

(b)

Figure 1: (a) $h(t)$ and (b) $\left\{h\left(t-i T_{C}\right), i=0,1, \ldots, N-1\right\}(N=16)$.

Here, $\Delta f \geq 1 / T_{S}$ to ensure orthogonality (separability) between subcarriers of the chip shape. The magnitude of $h(t)$ is shown in the Figure 1a assuming $\Delta f=1 / T_{S}$ and the set $\left\{h\left(t-i T_{C}\right), i=0,1, \ldots, N-1\right\}$ is shown in Figure $1 \mathrm{~b}$. It is important to note that $h\left(t-j T_{C}\right)$ and $h\left(t-k T_{C}\right)(k \neq j)$ are orthogonal to one another, and hence chips are separable. Referring to (3) and Figure 1, the CI chip shape corresponds to a frequency sampled version of the $\operatorname{sinc}(\cdot)$ shape.

\subsection{Proposed antenna array structure}

The CI/DS-CDMA signal characterized by (1), (2), and (3) is fed into a single $M$-element antenna array (see Figure 2). The $m$ th array element applies the phase shift $m \theta(t, \phi), m \in$ $[0, M-1]$.

The beam pattern oscillation is created by careful selection of the antenna element's phase offset, $\theta(t, \phi)$. The antenna array's beam pattern movement will be designed to ensure (1) constant large scale fading, that is, the mean of the Rayleigh fading is constant over the symbol duration $T_{S}$; and (2) that $L$ independent fades are generated within each partition $T_{S}$. In other words, the antenna array beam pattern is swept in a manner which ensures constant large scale fading over symbol duration $T_{S}$ while ensuring $L$ independent fades within each $T_{S}$.

\section{Criterion 1: Constant large-scale fading}

To ensure constant large-scale fading over each symbol time duration $T_{S}$, the beam pattern must remain in the antenna array HPBW. That is [4]

$$
\left|T_{S} \cdot \frac{d \phi}{d t}\right|=\kappa \cdot \text { HPBW }, \quad 0<\kappa<1,
$$

where $\phi$ is the azimuth angle, $d \phi / d t$ is the rate of antenna pattern movement, and $T_{S} \cdot(d \phi / d t)$ is the amount of antenna pattern movement in $T_{S}$. The parameter $\kappa$, selected such that $0<\kappa<1$, guarantees that the received antenna pattern amplitude is within the $3 \mathrm{~dB}$ beamwidth for the entire symbol duration. This parameter $\kappa$ is referred to throughout as the antenna array control parameter as it determines (restricts) the amount of beam pattern movement permitted in time duration $T_{S}$. Equation (4) corresponds to selecting the phase

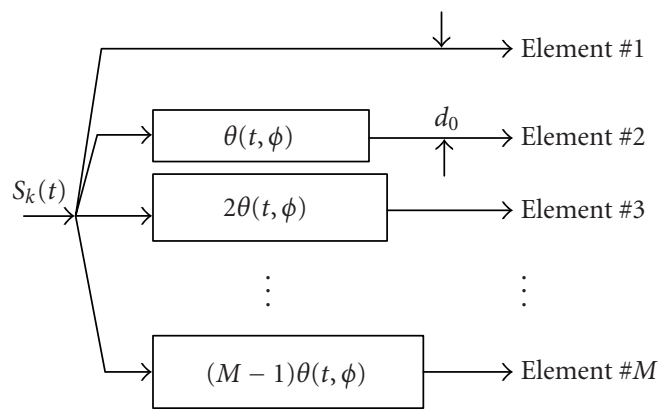

Figure 2: The antenna array structure over which the CI/DSCDMA signal is sent.

offset applied to the antenna elements according to [4]

$$
\begin{aligned}
& \theta(t, \phi)= \kappa \cdot \frac{2 \pi d_{0} \cdot|\sin (\phi)| \cdot \text { HPBW }}{\lambda_{0} T_{S}} \\
& \cdot\left(t-\frac{T_{S}}{2}\right), \quad t \in\left[0, T_{S}\right],
\end{aligned}
$$

where $d_{0}$ is the distance between the adjacent antenna elements as shown in Figure $2, \lambda_{0}$ is the average wavelength applied to the antenna array, and, for ease in presentation, we have assumed a mobile located at $\phi_{0} \approx \pi / 2$. After each time duration $T_{S}, \theta(t, \phi)$ returns to its $t=0$ value (returning the beam pattern to its original position) and $\theta(t, \phi)$ then recreates an identical spatial movement over the next $T_{S}$ duration. Assuming a small HPBW, (5) can be simplified to

$$
\theta(t) \cong \kappa \cdot \frac{2 \pi d_{0} \cdot \mathrm{HPBW}}{\lambda_{0} T_{S}} \cdot\left(t-\frac{T_{S}}{2}\right), \quad t \in\left[0, T_{S}\right] .
$$

\section{Criterion 2: Independent fades}

Movement of the antenna array beam pattern based on the time-varying antenna array phases in (5) or (6) results in a time varying channel, and rate of variation of the channel is measured by coherence time, $T_{C}$. Coherence time, in turn, determines the number of independent fades over duration $T_{S}$. Computation of the coherence time requires modeling a 
channel in the presence of a moving beam pattern. In $[5,6]$ a linear time-varying impulse response model is introduced to characterize the channel with a beam pattern oscillation based on (6). The channel impulse response was characterized using the so-called geometric-based stochastic channel model (GSCM) [14, 15]. Simulation results with the antenna array control parameter restricted to $0.0005<\kappa<0.05$ demonstrated that the channel coherence time due to beam pattern movement leads to an available diversity gain of up to $L \approx 7[5,6]$.

Specifically, based on our earlier work in $[5,6]$, we demonstrated how a 7-fold time diversity benefit is achieved (by beam pattern movement) in a mid-sized city center assuming three scatterers with an average size of $20 \mathrm{~m}$ in every $1000 \mathrm{~m}^{2}, \kappa=0.05$ (i.e., beam pattern movement corresponds to $5 \%$ of the HPBW), BS-mobile distance $x_{0}=1000 \mathrm{~m}$, and $\mathrm{HPBW}=0.3$ radian (i.e., $\mathrm{HPBW} \approx 17^{\circ}$ ). (To create $\mathrm{HPBW} \approx 17^{\circ}$ requires $M=6$ antenna array elementshence, the number of elements may be a small value.)

\section{RECEIVER DESIGN}

User $k$ 's signal, input to the antenna array of Figure 2, corresponds to (using (1) and (2))

$$
s_{k}(t)=\operatorname{Re}\left\{b_{k} \cdot e^{j 2 \pi f_{0} t} \cdot \sum_{i=0}^{N-1} c_{k}^{i} \cdot h\left(t-i T_{C}\right) \cdot g(t)\right\},
$$

which, using $h(t)$ in (3), leads to

$$
\begin{gathered}
s_{k}(t)=\operatorname{Re}\left\{b_{k} \cdot e^{j 2 \pi f_{0} t} \cdot \sum_{i=0}^{N-1} c_{k}^{i} \cdot \sum_{n=0}^{N-1} e^{j 2 \pi n \Delta f\left(t-i T_{C}\right)} \cdot g(t)\right\}, \\
s_{k}(t)=b_{k} \cdot g(t) \cdot\left(\sum_{i=0}^{N-1} c_{k}^{i} \cdot \sum_{n=0}^{N-1} \cos \left(2 \pi\left(f_{0}+n \Delta f\right) \cdot t-\beta_{n}^{i}\right)\right), \\
t \in\left[0, T_{S}\right],
\end{gathered}
$$

where $\beta_{n}^{i}=2 \pi i \cdot n \Delta f T_{C}$. The output of the $m$ th element of the antenna array, after application of phase offset $m \theta(t)$, is simply

$$
\begin{aligned}
s_{k}^{m}(t)= & b_{k} \cdot g(t) \\
& \cdot\left(\sum_{i=0}^{N-1} c_{k}^{i} \cdot \sum_{n=0}^{N-1} \cos \left(2 \pi\left(f_{0}+n \Delta f\right) \cdot t-\beta_{n}^{i}+m \theta(t)\right)\right) .
\end{aligned}
$$

The presence of $\theta(t)$ creates a frequency offset; however, with $\theta(t)$ selected according to (6) (and considering $\kappa<0.05)$, and assuming the distance between the antenna elements $d=\lambda / 2$, antenna HPBW $=0.3$, and $M=6$ elements, it is easily shown that the frequency offset induced by $\theta(t)$ is less than $5 \%$ of a $1 \mathrm{MHz}$ bandwidth. Hence, we ignore this frequency offset in our presentation. The total downlink transmitted signal, considering all antenna elements (all $\mathrm{m}$ ) and all users (all $k)$ in $t \in\left[0, T_{S}\right]$, is (from (9))

$$
\begin{gathered}
s(t)=\sum_{k=1}^{K} b_{k} \cdot g(t) \cdot\left(\sum _ { i = 0 } ^ { N - 1 } c _ { k } ^ { i } \cdot \sum _ { n = 0 } ^ { N - 1 } \frac { 1 } { M } \cdot \sum _ { m = 0 } ^ { M - 1 } \operatorname { c o s } \left(2 \pi\left(f_{0}+n \Delta f\right)\right.\right. \\
\left.\left.\cdot t-\beta_{n}^{i}+m \theta(t)\right)\right), \quad t \in\left[0, T_{S}\right],
\end{gathered}
$$

where $1 / M$ is a normalization factor compensating for transmission over $M$ array elements.

At the receiver side, the transmit diversity (due to antenna array movement generated by $\theta(t)$ ) corresponds to an $L$-fold time diversity. Hence, the received signal in duration $\left[0, T_{S}\right]$ can be divided into time slots $\left[l T_{S} / L,(l+1) T_{S} / L\right]$, where $l \in[0, L-1]$, and each time slot contains a signal with an independent frequency-selective fade. The received signal corresponds to

$$
\begin{gathered}
r^{l}(t)=\sum_{k=1}^{K} b_{k} \cdot g(t) \cdot\left(\sum_{i=0}^{N-1} c_{k}^{i} \cdot \sum_{n=0}^{N-1} \alpha_{n}^{l} \cdot A F(t, \phi)\right. \\
\cdot \cos \left(2 \pi\left(f_{0}+n \Delta f\right) \cdot t-\beta_{n}^{i}\right. \\
\left.\left.+\frac{M-1}{2} \gamma(t, \phi)+\xi_{n}^{l}\right)\right)+n^{l}(t), \\
t \in\left[\frac{l T_{S}}{L}, \frac{(l+1) T_{S}}{L}\right], l=0,1, \ldots, L-1
\end{gathered}
$$

First, we explain the $\alpha_{n}^{l}$ and $\xi_{n}^{l}$ terms in (11). Because each chip shape is a multicarrier signal, the frequency selectivity of the fade is resolved by the multicarrier components (as in OFDM [16] and MC-CDMA [17]). That is, each carrier $n$, $n \in\{0,1, \ldots, N-1\}$, that makes up the chip shape, experiences a unique flat fade. $\alpha_{n}^{l}$ is the fade on the $n$th carrier in the $l$ th time slot (due to fading) and $\xi_{n}^{l}$ is the phase offset in the $n$th carrier and $l$ th time slot (due to fading) (hereafter, this phase is assumed to be tracked and removed). The fades $\alpha_{n}^{l}$ over the subcarriers that make up each CI chip, that is, $\left\{\alpha_{0}^{l}, \alpha_{1}^{l}, \ldots, \alpha_{N-1}^{l}\right\}$ are correlated Rayleigh random variables with correlation coefficient between the $p$ subcarrier fade and the $q$ subcarrier fade characterized by [18]

$$
\rho_{p, q}=\frac{1}{1+\left((p-q) \cdot\left(\Delta f /(\Delta f)_{C}\right)\right)^{2}},
$$

where $(\Delta f)_{C}$ is the coherence bandwidth of the channel. In addition, in (11), the $n^{l}(t)$ term represents the white Gaussian noise in the $l$ th time slot, and the antenna array introduces the phase offset $\gamma(t, \phi)$ corresponding to

$$
\gamma(t, \phi)=\left(\frac{2 \pi d_{0}}{\lambda_{0}}\right) \cdot \cos \phi+\theta(t)
$$

Here, $\left(2 \pi d_{0} / \lambda_{0}\right) \cdot \cos \phi$ represents the phase offset due to the difference in distance between antenna array elements and the mobile (assuming the smart antenna array is mounted horizontally). Moreover, in (11), the antenna array also 


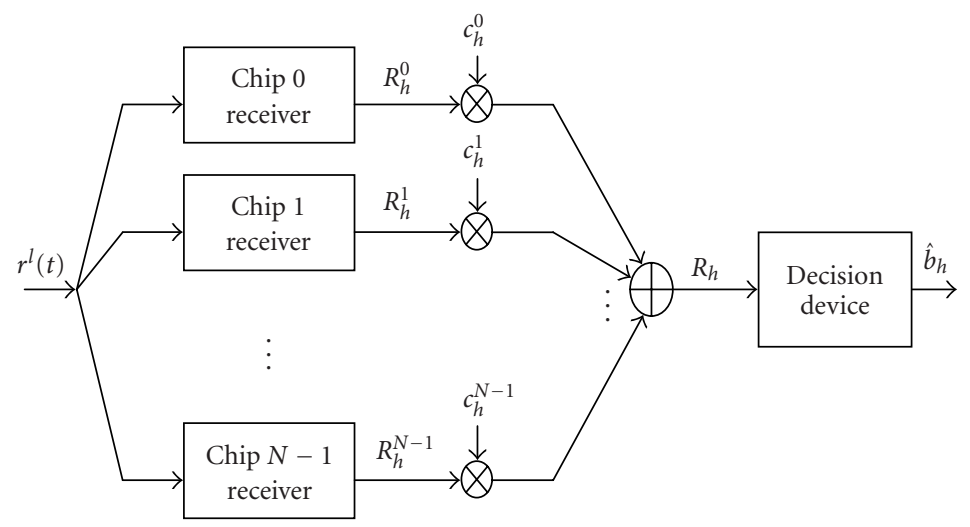

(a)

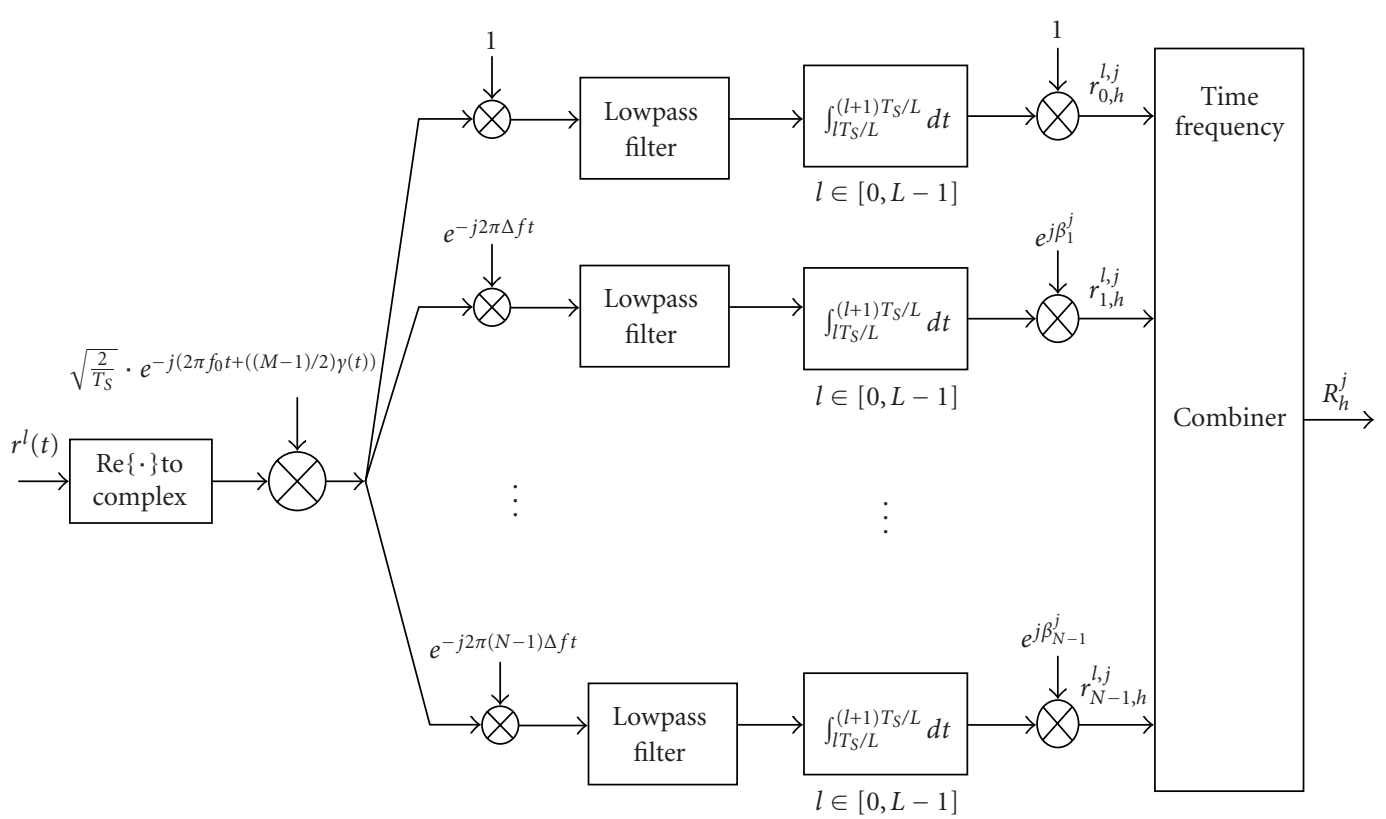

(b)

FIGURE 3: User $h$ (a) mobile receiver and (b) chip $j$ receiver.

introduces the normalized gain $A F(t, \phi)$, corresponding to

$$
A F(t, \phi)=\frac{1}{M} \cdot\left[\frac{\sin ((M / 2) \gamma(t, \phi))}{\sin ((1 / 2) \gamma(t, \phi))}\right] .
$$

Assuming the mobile is located on the antenna beam main axis $\left(\phi_{0}=\pi / 2\right)$, and assuming a small beam pattern movement $(\kappa<0.05)$, we can simplify (11) by assuming $\gamma(t, \phi) \cong$ $\gamma(t)=\theta(t)$ and $A F(t, \phi) \cong 1$ at the mobile's position for all $t \in\left[0, T_{S}\right]$.

The CI/DS-CDMA receiver is shown in Figures $3 a$ and $3 \mathrm{~b}$, where Figure $3 \mathrm{a}$ shows the overall receiver structure for user $h$, and Figure 3 b details the block entitled "chip $j$ receiver" (in Figure 3a). In other words, the receiver operates as follows: first, the received signal is processed through a total of $N$ chip receivers, where chip $j$ 's receiver (a) decomposes its chip into $N$ carrier components, and (b) recombines across the carrier components to recreate the chip while achieving a frequency diversity benefit. In addition, because a time diversity benefit is available (via transmit diversity), chip $j$ 's receiver also (c) combines across time components to recreate the chip with a frequency-time diversity gain. Next, once each chip is recreated with an enhanced diversity benefit, the receiver of Figure 3a performs a combining across chips in a usual DS-CDMA manner to eliminate interfering users' signals.

Mathematically, the receiver operates as follows. First, the received signal enters chip $j$ 's receiver. Here, the carrier component is removed from the incoming signal, and the signal is split into $N$ branches (one per carrier component). On the $n$th branch, the $n$th carrier is returned to baseband and separated from other carriers by application of a lowpass filter. 
(To ensure perfect separability of the carriers (that make up the $j$ th chip) via filtering, we select $\Delta f=2 / T_{S}$.)

Each baseband signal (one per carrier) is integrated over each interval over which the fade is constant, that is, over $t \in$ $\left[l T_{S} / L,(l+1) T_{S} / L\right], l \in[0, L-1]$. After applying phase offsets to the $N$ frequency components (phase offsets corresponding to the delay $j T_{C}$, separating the $j$ th chip from other chips), the signal in the $j$ th chip's receiver, for each carrier $n, n \in$ $[0, N-1]$, and time interval $l, l \in[0, L-1]$, corresponds to

$$
\begin{aligned}
r_{n, h}^{l, j}= & \frac{1}{L} \sqrt{E_{S}} \cdot \alpha_{n}^{l} \cdot b_{h} \cdot c_{h}^{j}+\frac{1}{L} \sqrt{E_{S}} \cdot \alpha_{n}^{l} \cdot b_{h} \cdot \sum_{\substack{i=0 \\
i \neq j}}^{N-1} c_{h}^{i} \cdot \rho_{n}^{i, j} \\
& +\frac{1}{L} \sqrt{E_{S}} \cdot \alpha_{n}^{l} \cdot \sum_{\substack{k=1 \\
k \neq h}}^{K} c_{k}^{j} \cdot b_{k} \\
& +\frac{1}{L} \sqrt{E_{S}} \cdot \alpha_{n}^{l} \cdot\left(\sum_{\substack{k=1 \\
k \neq h}}^{K} b_{k} \cdot \sum_{\substack{i=0 \\
i \neq j}}^{N-1} c_{k}^{i} \cdot \rho_{n}^{i, j}\right)+n_{n, h}^{l, j},
\end{aligned}
$$

where $\sqrt{E_{S}}=\sqrt{T_{S} / 2}$. In (15), the first term represents the desired $n$th frequency component and $l$ th time component of chip $j$ for the desired user (user $h$ ); the second term is the interchip interference due to other chips from the same user (where $\rho_{n}^{i, j}=\cos \left(\beta_{n}^{i}-\beta_{n}^{j}\right.$ ) is the correlation between the $i$ th chip and the $j$ th chip in carrier $n$ ); the third term is the interference due to the same chip from other users, and the fourth term represents the interference from different chips of different users. Moreover, $n_{n, h}^{l, j}$ is a zero mean Gaussian random variable with variance $\left(N_{0} / 2\right) /\left(N^{2} \cdot L\right)$, independent across different carriers $n$ and different time slots $l$, but correlated across chips, with correlation $\rho_{n}^{i, j}$ between the $i$ th chip noise and the $j$ th chip noise.

It is also important to note the factor of $1 / L$ in the first term (desired term) in (15), which is a direct result of the division of the received signal interval into $L$ partitions (to create $L$-fold time diversity) (i.e., a direct result of the $L$-fold oversampling strategy).

Following the decomposition of the $j$ th chip into its time and frequency components, each with a unique fade, a linear combining strategy is employed to recreate the $j$ th chip with a joint time-frequency diversity benefit. Using the linear combining scheme discussed in the next paragraph, we combine the $r_{n, h}^{l, j}$ over time components $(l)$ and frequency components $(n)$ (with $L \times N$ diversity components, $L$ over time and $N$ over frequency) to simultaneously reduce the interchip interference and the noise, and achieve high diversity gains. This leads to the output, $R_{h}^{j}$, which (referring to Figure $3 a$ ) is combined across the $N$ chips in the usual DSCDMA manner to eliminate other users interference (term 3 in $(15))$. The chip combiner output for user $h$ corresponds to

$$
R_{h}=\sum_{j=0}^{N-1} c_{h}^{j} \cdot R_{h}^{j}
$$

This $R_{h}$ term enters a hard decision device which generates the final decision, $\hat{b}_{h}$. The time-frequency combiner recreating the $j$ th chip from its time-frequency components (in Figure $3 \mathrm{~b}$ ) is designed using EGC-MMSEC, that is, EGC across $L$ time components followed by MMSEC across $N$ frequency components. Applying EGC in time, then the Wienner filter principle [19] to determine the MMSEC across carriers, the decision variable corresponds to

$$
R_{h}^{j}=\sum_{n=0}^{N-1} \frac{\sqrt{E_{S}} \cdot \alpha_{n} / L}{P \cdot K \cdot\left(\alpha_{n}\right)^{2}+N_{0 n}^{\prime} / 2} \cdot r_{n, h}^{j},
$$

where

$$
\begin{aligned}
& r_{n, h}^{j}=\sum_{l=0}^{L-1} r_{n, h}^{l, j}=\frac{1}{L} \sqrt{E_{S}} \cdot b_{h} \cdot c_{h}^{j} \cdot \alpha_{n} \\
& +\frac{1}{L} \sqrt{E_{S}} \cdot b_{h} \cdot \alpha_{n} \cdot \sum_{\substack{i=0 \\
i \neq j}}^{N-1} c_{h}^{i} \cdot \rho_{n}^{i, j} \\
& +\frac{1}{L} \sqrt{E_{S}} \cdot \alpha_{n} \cdot \sum_{\substack{k=1 \\
k \neq h}}^{K} c_{k}^{j} \cdot b_{k} \\
& +\frac{1}{L} \sqrt{E_{S}} \cdot \alpha_{n} \cdot\left(\sum_{\substack{k=1 \\
k \neq h}}^{K} b_{k} \cdot \sum_{\substack{i=0 \\
i \neq j}}^{N-1} c_{k}^{i} \cdot \rho_{n}^{i, j}\right)+n_{n, h}^{j}, \\
& \alpha_{n}=\sum_{l=0}^{L-1} \alpha_{n}^{l} \\
& n_{n, h}^{j}=\sum_{l=0}^{L-1} n_{n, h}^{l, j} \\
& P=\frac{E_{S}}{L^{2}} \cdot \begin{cases}N & \text { for } n=0 \text { or } \frac{N}{2}, \\
\frac{N}{2} & \text { else, }\end{cases}
\end{aligned}
$$

and $N_{0 n}^{\prime} / 2$ is the noise variance of $n_{n, h}^{j}$ in (20), that is, $\left(N_{0} / 2\right) / N^{2}$.

\section{SIMULATED PERFORMANCE}

For simulation purposes, we consider (1) CI/DS-CDMA with a processing gain of $N=32$; (2) each CI/DS-CDMA chip is composed of $N=32$ carriers (see (3)); (3) the CI/DS-CDMA system is fully loaded with $K=32$ orthogonal users employing Hadamard-Walsh codes; (4) the frequency selectivity of the channel results in 4-fold frequency diversity over the entire bandwidth, that is, $(\Delta f)_{C} / B W=0.25$, and (5) beam pattern movement results in $L=7$ independent fades in the duration $T_{S}$ (see Section 2 and [5]).

In Figure 4, a typical simulation result is provided for the proposed CI/DS-CDMA—oscillating beam antenna-array merger. The simulation results in Figure 4 are compared with those of CI/DS-CDMA with a conventional smart antenna at the BS. Here, MMSEC is applied to the subcarriers of the received CI/DS-CDMA signal $[9,10,11,12,13]$. It is observed that the introduction of a smart antenna array with 


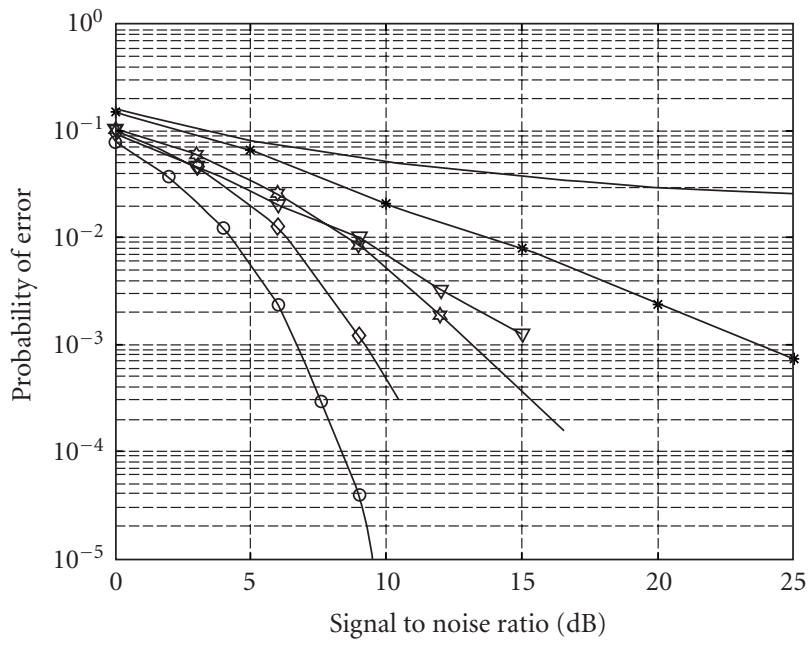

- Traditional DS-CDMA without beam oscillation

* CI/DS-CDMA without beam oscillation

CI/DS-CDMA with beam oscillation, EGC-EGC

$\nabla$ CI/DS-CDMA with beam oscillation, EGC-MRC

$\diamond$ CI/DS-CDMA with beam oscillation, EGC-MMSEC

๑- AWGN channel

FIGURE 4: Simulation results.

beam-pattern oscillation at the BS introduces an improvement of more than $14 \mathrm{~dB}$ at a probability of error of $10^{-3}$ (at the mobile via EGC-MMSEC combining technique) compared to CI/DS-CDMA with traditional smart antenna arrays. When compared to traditional DS-CDMA with RAKE reception (e.g., [8]) combined with a conventional smart antenna array, even larger performance gains are achieved, as shown in Figure 4. In Figure 4, EGC-MMSEC technique is compared with EGC-EGC and EGC-MRC (maximal ratio combining) schemes. EGC-MMSEC achieves excellent performance relative to other combining options.

\section{DISCUSSION AND CONCLUSION}

CI/DS-CDMA signals are sent via a single antenna array at the $\mathrm{BS}$, and received by a single antenna at the mobile station. The phase shifts introduced to the BS antenna array elements are designated to control the antenna pattern movement (oscillation) such that it achieves directionality and transmit diversity.

Receivers employ diversity combining in the frequency and time domains, and significant performance improvement is shown when compared to the CI/DS-CDMA system with a conventional antenna array. This performance gain highlights the significance of small beam pattern movement in smart-antenna-array CDMA systems. This performance leads to high network capacity in terms of number of users.

\section{ACKNOWLEDGMENT}

This work was supported by NASA Phase II SBIR Grant: "Development of a Wireless Communication System to Support Airport Surface Operations."

\section{REFERENCES}

[1] A. Hiroike, F. Adachi, and N. Nakajima, "Combined effects of phase sweeping transmitter diversity and channel coding," IEEE Trans. Veh. Technol., vol. 41, no. 2, pp. 170-176, 1992.

[2] V. Tarokh, N. Seshadri, and A. R. Calderbank, "Space-time codes for high data rate wireless communication: performance criterion and code construction," IEEE Trans. Inform. Theory, vol. 44, no. 2, pp. 744-765, 1998.

[3] S. A. Zekavat, C. R. Nassar, and S. Shattil, "Combined directionality and transmit diversity via smart antenna spatial sweeping," in Proc. 38th Annual Allerton Conference on Communication, Control, and Computing, pp. 203-211, University of Illinois at Urbana-Champaign, Ill, USA, October 2000.

[4] S. A. Zekavat, C. R. Nassar, and S. Shattil, "Oscillatingbeam smart antenna arrays and multicarrier systems: achieving transmit diversity, frequency diversity, and directionality," IEEE Trans. Veh. Technol., vol. 51, no. 5, pp. 1030-1039, 2002.

[5] S. A. Zekavat and C. R. Nassar, "Geometric-based stochastic channel modeling for adaptive antennas with oscillating beam patterns," in Proc. 12th IEEE International Symposium on Personal, Indoor and Mobile Radio Communications, vol. 1, pp. 130-134, San Diego, Calif, USA, September 2001.

[6] S. A. Zekavat and C. R. Nassar, "Smart antenna arrays with oscillating beam patterns: characterization of transmit diversity in semi-elliptic coverage," IEEE Trans. Communications, vol. 50, no. 10, pp. 1549-1556, 2002.

[7] A. J. Viterbi, CDMA. Principles of Spread Spectrum Communication, Addison-Wesley Publishing, Reading, Mass, USA, 1995.

[8] U. S. Goni and A. M. D. Turkmani, "BER performance of a direct-sequence CDMA system in multipath fading mobile radio channels with Rake reception," in IEEE 44th Vehicular Technology Conference, vol. 2, pp. 747-751, Stockholm, Sweden, June 1994.

[9] C. R. Nassar, B. Natarajan, and Z. Wu, "Multi-carrier technology platform for wireless communications. Part 1: Highperformance, high-throughput TDMA and DS-CDMA via multi-carrier implementations," to appear in Wireless Communications and Mobile Computing.

[10] Z. Wu, C. R. Nassar, and S. Shattil, "High performance DSCDMA via carrier interferometry," in Wireless 2001, pp. 564569, Calgary, Alberta, July 2001.

[11] Z. Wu, C. R. Nassar, and S. Shattil, "Chip shaping advances for high capacity DS-CDMA," in 2001 International Conference on Third Generation Wireless and Beyond, pp. 928-932, San Francisco, Calif, May-June 2001.

[12] Z. Wu and C. R. Nassar, "MMSE frequency combining for CI/DS-CDMA," in IEEE Radio and Wireless Conference, pp. 103-106, Denver, Colo, USA, 2000.

[13] C. R. Nassar and Z. Wu, "High performance broadband DSCDMA via carrier interferometry chip shaping," in International Symposium on Advanced Radio Technologies, Boulder, Colo, USA, September 2000.

[14] R. B. Ertel, P. Cardieri, K. W. Sowerby, T. S. Rappaport, and J. H. Reed, "Overview of spatial channel models for antenna array communication systems," IEEE Personal Communications, vol. 5, no. 1, pp. 10-22, 1998.

[15] A. F. Molisch, A. Kuchar, J. Laurila, K. Hugl, and E. Bonek, "Efficient implementation of a geometry-based directional model for mobile radio channels," in IEEE 50th Vehicular Technology Conference, vol. 3, pp. 1449-1453, Amsterdam, Netherlands, 1999.

[16] R. van Nee and P. Ramjee, OFDM for Wireless Multimedia Communications, Artech House Publishers, Boston, Mass, USA, 2000. 
[17] S. Hara and R. Prasad, "Overview of multicarrier CDMA," IEEE Communications Magazine, vol. 35, no. 12, pp. 126-133, 1997.

[18] W. C. Jakes, Microwave Mobile Communications, John Wiley \& Sons, New York, NY, USA, 1974.

[19] S. Haykin, Adaptive filter theory, Prentice-Hall, Englewood Cliffs, NJ, USA, 2nd edition, 1991.

Seyed Alireza Zekavat received his Ph.D. degree in electrical and computer engineering from Colorado State University, Colo, USA in 2002. From 1993 to 1998 he was with Civil Aviation College of Technology, Tehran, Iran. Since August 2002 he has been with Michigan Technological University (MTU), Houghton, Mich, USA. His research interests include wireless communications, radar theory, statistical modeling, adaptive beam forming, and neural networks. He is the founder and the director of the laboratory for wireless communication research in MTU. He has published about 40 journal and conference papers and has coauthored the book Multi-Carrier Technologies for Wireless Communications.

Carl R. Nassar received his B.S., M.S., and Ph.D. degrees from McGill University in 1989, 1990, and 1997, respectively. Between his M.S. and Ph.D. degrees, Dr. Nassar worked for a time as a design engineer at CAE Electronics in Montreal, Canada. Upon completion of his Ph.D., he spent a year as an Assistant Professor at McGill University. Soon thereafter, he headed for the hills of Colorado, where he has been an as-

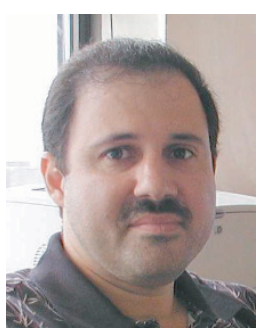
sistant professor for the past five years. Dr. Nassar is the Director of Colorado State University's RAWCom (Research in Advanced Wireless) laboratory, a position which enables him to pursue his interest in wireless telecommunications. With funding from the NSF, NASA, industry, and the State of Colorado, Dr. Nassar's research focuses on the design of high network capacity, high QOS multipleaccess technologies. In particular, Dr. Nassar focuses on advances in multicarrier technologies such as MC-CDMA and OFDM, and seeks a common multicarrier platform for all wireless multiple access based on his proposed CI technology. Dr. Nassar's work has been published in over 90 international conference proceedings and journal articles, and he is the author of two books: Telecommunications Demystified, a friendly engineering look at telecommunication systems, and Multi-Carrier Technologies for Future Generation Wireless.

Steve Shattil is the Chief Scientist at CIAN Systems Inc., where he is leading development in carrier interferometry and other coding technologies. Mr. Shattil has over 15 -year experience in the wireless industry. Prior to founding CIAN, he was Founder and Chief Technical Officer of Genesis Telecom. Genesis invented highly bandwidthefficient antenna array technologies for broadband wireless communications. Over the last decade, Mr. Shattil has led the development of baseband and RF processors for broadband products, and is world renowned in both academia and industry for his innovation, leadership, and
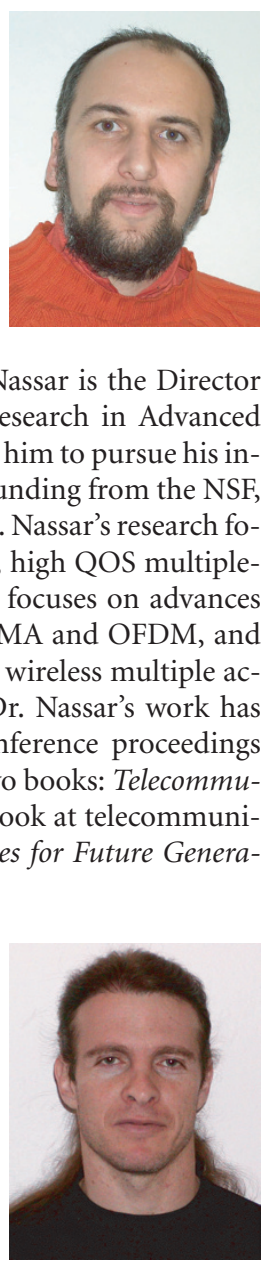

the advancement of wireless communications. Mr. Shattil has authored dozens of domestic and international patents. He holds an M.E. in electrical engineering from University of Colorado where he pioneered advances in high-data-rate signal processing. Mr. Shattil also holds an M.S. in physics from Colorado School of Mines, where he advanced the field of laser physics and built the first laser that generates carrier interferometry signals. Mr. Shattil holds a B.S. in physics from Rensselaer Polytechnic Institute. 